\title{
Three-dimensional vortex flow field around a circular cylinder in a quasi-equilibrium scour hole
}

\author{
SUBHASISH DEY \\ Department of Applied Mechanics, Regional Engineering College, \\ Durgapur 713209 , India
}

MS received 3 November 1994; revised 7 July 1995

\begin{abstract}
The three-dimensional flow field around a circular cylinder in a quasi-equilibrium scour hole under a clear water regime has been investigated experimentally. The distribution patterns of tangential, radial and vertical components of velocity upstream and longitudinal component of velocity downstream of a cylinder have been presented in this paper. The flow simulation results show the existence of a helical motion of fluid around the cylinder.
\end{abstract}

Keywords. Three-dimensional flow; hydrodynamics; vortex flow; scouring; flow around cylinder; velocity profiles.

\section{Introduction}

A cylinder, embedded vertically in a loose bed, causes obstruction to form a threedimensional separation of boundary layer flow and is conducive to the development of a localized scour. As a result of the interaction between the two-dimensional approaching boundary layer flow and the adverse pressure gradient set by the cylinder, the problem becomes complex. Detailed study of the general flow pattern for such a case has been reported by Hjorth (1975), Melyille (1975), and Dargahi (1987). The components of the flow field are the downflow, horseshoe vortex, wake vortex and bow wave, as shown schematically in figure 1 . The approaching flow gradually becomes zero along the vertical line in the front face of the cylinder. Since the profile of the approaching velocity along the vertical decreases downward, starting with a maximum at the free surface and becoming zero at the bed, the stagnation pressure gradient is negative downward. Thus, the flow is driven down in the close front of the cylinder. The horseshoe vortex, developed due to the separation of flow at the edge of the scour hole upstream, rolls to form a large helical flow which migrates along the sides of the cylinder downstream (Raudkivi 1986). A bow wave is formed at the upstream free surface adjacent to the cylinder, rotating in the opposite direction of the horseshoe vortex. The stagnation pressure also accelerates the flow past the cylinder, resulting in flow separation at the sides of the cylinder and creating the wake with the cast off vortices at the interfaces to the main flow. Enhanced 

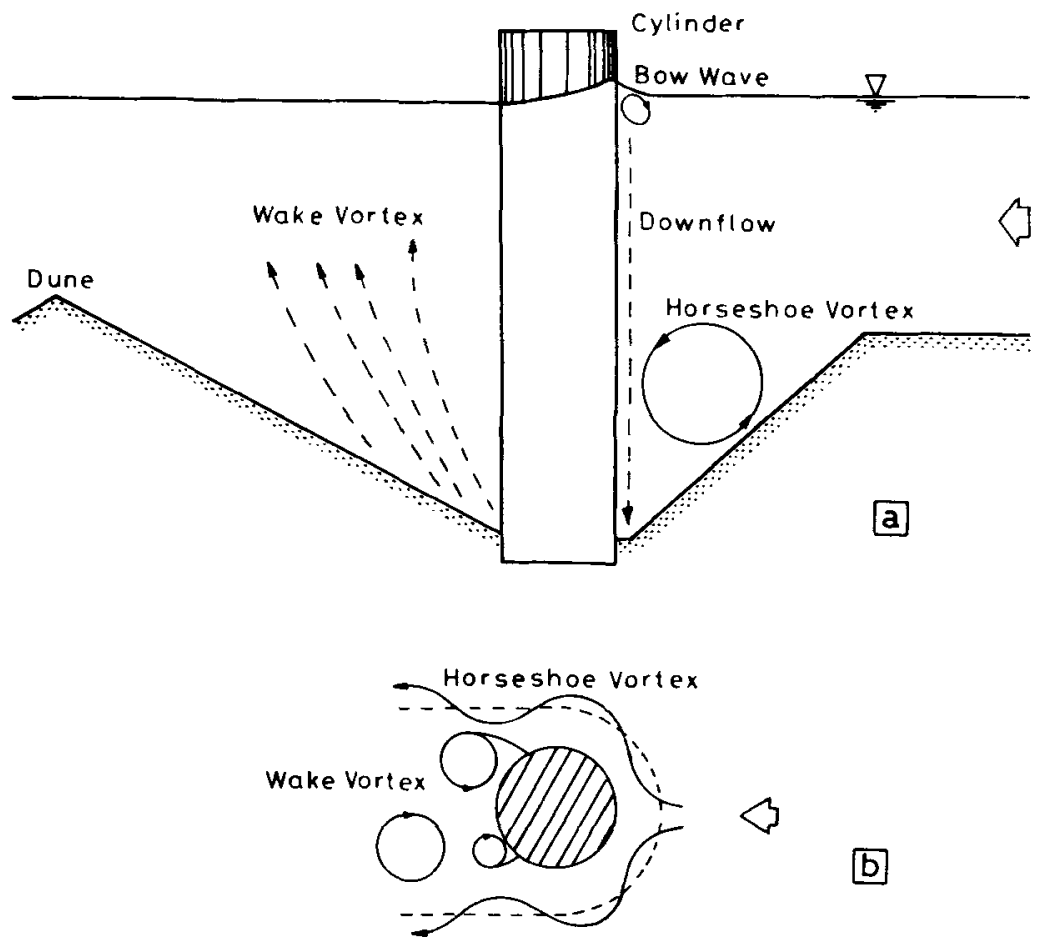

b

Figure 1. Definition sketch.

velocities in front of and to the sides of a cylinder scour the loose bed and carve out $U$-shaped scour hole with open ends of the U directing downstream.

Depending on the supply of sediment by the approaching flow, localized scour occurs in two ways. Clear water scour refers to the situation where no sediment is supplied by the approaching flow into the scour zone. Live bed scour, in contrast, occurs where there is a general transport of sediment by the approaching flow to the zones subjected to scour. Summary pertaining to the work on local scouring has been comprehensively reviewed by Breusers et al (1977) and Dargahi (1982). Although the characteristic features of the general flow structure around a circular cylinder in the scour zone have been documented, the nature of the distribution patterns of the velocity components seems to have received little attention so far.

The aim of the present experimental investigation is to determine the behavioural patterns of the distribution of velocity components of the three-dimensional vortex flow field around a circular cylinder in a quasi-equilibrium scour hole under a clear water regime. The experimental data are utilized to obtain the flow simulation results.

\section{Experimentation}

\subsection{Experimental set-up and programme}

The experiments were conducted in a $1.3 \mathrm{~m}$ long, $0.81 \mathrm{~m}$ wide and $0.25 \mathrm{~m}$ deep test section located $6 \mathrm{~m}$ away from the inlet of a $10 \mathrm{~m}$ long flume. A false floor was 
constructed along the length of the flume $0.25 \mathrm{~m}$ above the bottom, and uniformly graded fine sand of mean diameter $\left(d_{50}\right) 0-58 \mathrm{~mm}$ used for local scouring was glued along its surface. Geometric standard deviation $\left(\sigma_{g}\right)$ of the particle size distribution given by $\left(d_{84} / d_{16}\right)^{1 / 2}$ was 1.31 which was less than 1.4 for a uniformly graded sand (Dargahi 1987). A perspex circular cylinder of diameter $(b) 0.076 \mathrm{~m}$ was placed at the middle of the test section. Experiments were run under clear water scour at an approaching flow depth $(h)$ of $0.05 \mathrm{~m}$ and a mean approaching velocity $(U)$ of $0.261 \mathrm{~m} / \mathrm{s}$. Although a ratio $h / b$ up to 3 was found to be desirable for severe scouring, in this study $h / b$ was set to 0.66 to have the controlled flow in the flume. The flow field, to be measured, would however remain similar for higher values of $h / b$. The semi-logarithmic plot of the velocity at $2 \mathrm{~m}$ upstream from the cylinder centre, found to be free flow, was used to determine the shear velocity of approaching flow $\left(u_{*}\right)$. The magnitude of $u_{*}$ found to be $0.0169 \mathrm{~m} / \mathrm{s}$ was less than the critical bed shear velocity $u_{* c}$ for the initiation of particle motion i.e. $0.017 \mathrm{~m} / \mathrm{s}$. The experiments were started, setting the inflow rate by the inlet valve and maintaining the required flow depth by the downstream control. Run time for a period of $12 \mathrm{~h}$ was taken to ensure that the quasi-equilibrium state (discussed in the succeeding section) was reached. After $12 \mathrm{~h}$, the inflow rate was slowly reduced to zero and the water was carefully drained out from the scour hole.

\subsection{Bed stabilization}

A synthetic resin mixed with water (1:3 by volume) was sprayed on the scour bed when it was reasonably dry. The sand was sufficiently impregnated with the resin when it was left to set for $12 \mathrm{~h}$. The resin was polymerized by spraying a coat of dilute formic acid. After $48 \mathrm{~h}$, the bed profile became rock-hard, facilitating measurements.

\subsection{Measuring techniques}

The three components of velocity vectors were measured using a five-hole pitot sphere (Shukry 1950) which had a spherical sensing head of $8 \mathrm{~mm}$ diameter and five holes lay on two meridians perpendicular to each other. The appropriate calibration curves enabled the pitch angle and the velocity magnitude to be determined. The direction in the horizontal plane of velocity vector was determined by a protractor attached to the probe, equalizing pressures at the two pressure points opposed in plan. In the regions of negligible vertical velocity (at $75^{\circ}$ upstream and in the downstream), a three-tube yaw probe (Rajaratnam \& Muralidhar 1967) was used to measure the horizontal velocity vectors. This probe was made of three brush tubes of $3 \mathrm{~mm}$ OD and $1.8 \mathrm{~mm}$ ID, rigidly fixed together. The centre tube had a flat nose, while the side tubes were chamfered at an angle $45^{\circ}$. The downflow in the front face of the cylinder was measured using a specially made pitot tube because pitot sphere was unsuitable there. As there was no flow across the downflow i.e. the pressure gradient normal to the flow direction is zero, this probe was found to be useful. It had a vertical static tube with radial holes at the end and a stagnation tube facing the downflow, with $2 \mathrm{~mm}$ OD and $1 \mathrm{~mm}$ ID each. All the probes were connected to the precision manometers (inverted type) which had air at the top. The manometer reading up to $0.1 \mathrm{~mm}$ accuracy was measured by a Vernier attachment. The minimum velocity vector was registered to be $0.04 \mathrm{~m} / \mathrm{s}$. The flow 
direction and magnitude within the scour hole changed extensively making the measurement difficult. A light aluminium fin, known as direction finder, was attached to the end of a steel rod of $1 \mathrm{~mm}$ diameter. The fin could rotate freely about its axis to show the flow direction approximately when it was dipped in the flow. It was estimated, however, that the mean velocity directions were within $\pm 10^{\circ}$. The most difficult part in the flow measurement was to ascertain the reversed flow which was successfully carried out by the fin. To get the exact flow direction, the pitot sphere/yaw probe was placed at the appropriate position to face the flow and was rotated along a horizontal plane until it registered the same pressure in the side holes. Also, the points of detachment of the horseshoe vortex on both sides of the cylinder determined by the fin were at $\pm 75^{\circ}$. The flow field in the wake region was ill-defined and was beyond the realm of the study.

\subsection{Measurement errors and corrections}

The errors for pitot tube (Nwachukwu 1979) which may possibly occur in the measurements are: (i) the error in presence of a pitot tube near to the bed which results in higher impact of pressure record than the actual one due to shifting of streamlines; and (ii) the error due to the turbulent fluctuation. The error in the measured velocity near bed, based on $1 / 7$ th power law of velocity distribution, was found to be $7 \%$ (Tingsanchali \& Maheswaran 1990). Since no attempt has been made to quantify this type of instrumental error for a five-hole pitot sphere and three-tube yaw probe, it was assumed that these were subjected to the same error magnitude as that of the pitot tube. The error in the mean velocity due to turbulence was estimated from the relationship given by Goldstein (1983). Based on the turbulent intensity for equilibrium scour measured by Melville \& Raudkivi (1977), the local velocity was found to be overestimated by $2 \%$ (Dey 1991). So no correction was applied to suppress it. All the measurements were repeated with good accuracy until the two separate measurements at a point were registered to be equal. The errors of the measurements carried out in the reversed flow regions were usually higher than in other regions of the flow, which were taken with some reservation.

The approaching flow crossed the upper portion of the pitot sphere and the reversed flow near bed was affected. However, the correction factor, determined using a micropitot tube, was used for the data of velocity measurements. The velocities measured by a micro-pitot tube along the sloping bed, providing a free passage of approaching flow, were compared with those measured by the pitot sphere. The correction factors estimated at $0^{\circ}, 15^{\circ}$ and $30^{\circ}$ were $1 \cdot 18,1 \cdot 11$ and 1.05 respectively.

\section{Quasi-equilibrium scour}

The process of digging by the downflow at the cylinder base and the erosion of slant bed continued until a quasi-equilibrium state (Dey et al 1992b) was reached. This happens when the particles can no longer be dislodged by the impact of the downflow. In this state, the slant bed eroded continually in the form of a few rolling particles which were subsequently carried out by the arms of the horseshoe vortex, without any noticeable change in scour depth. Full equilibrium was reached, when the rolling motion was ceased, after an impractically long experimental time. The average angle of the upstream sloping bed, in the quasi-equilibrium state, was about $15 \%$ greater than the 
angle of repose $(\phi)$ of the bed sand in still water. The greater angle, maintained by the shear force of the reversed flow along the slope of the hole, may be termed "dynamic angle of repose" (Dey 1991; Dey et al 1992b). This was also evident in Melville \& Raudkivi (1977) and Jain \& Fischer (1979). In the present study the dynamic angle of repose was found to be $37^{\circ}$, with an upstream conical scour hole of depth $0.096 \mathrm{~m}$ below the original bed for the sand having $\phi$ of $32^{\circ}$.

\section{Simplified geometry of scour hole}

In the upstream, the azimuthal section of the quasi-equilibrium scour hole (figure 2) is divided into zone 1 i.e. vertically above the sloping bed and zone 2 i.e. vertically above
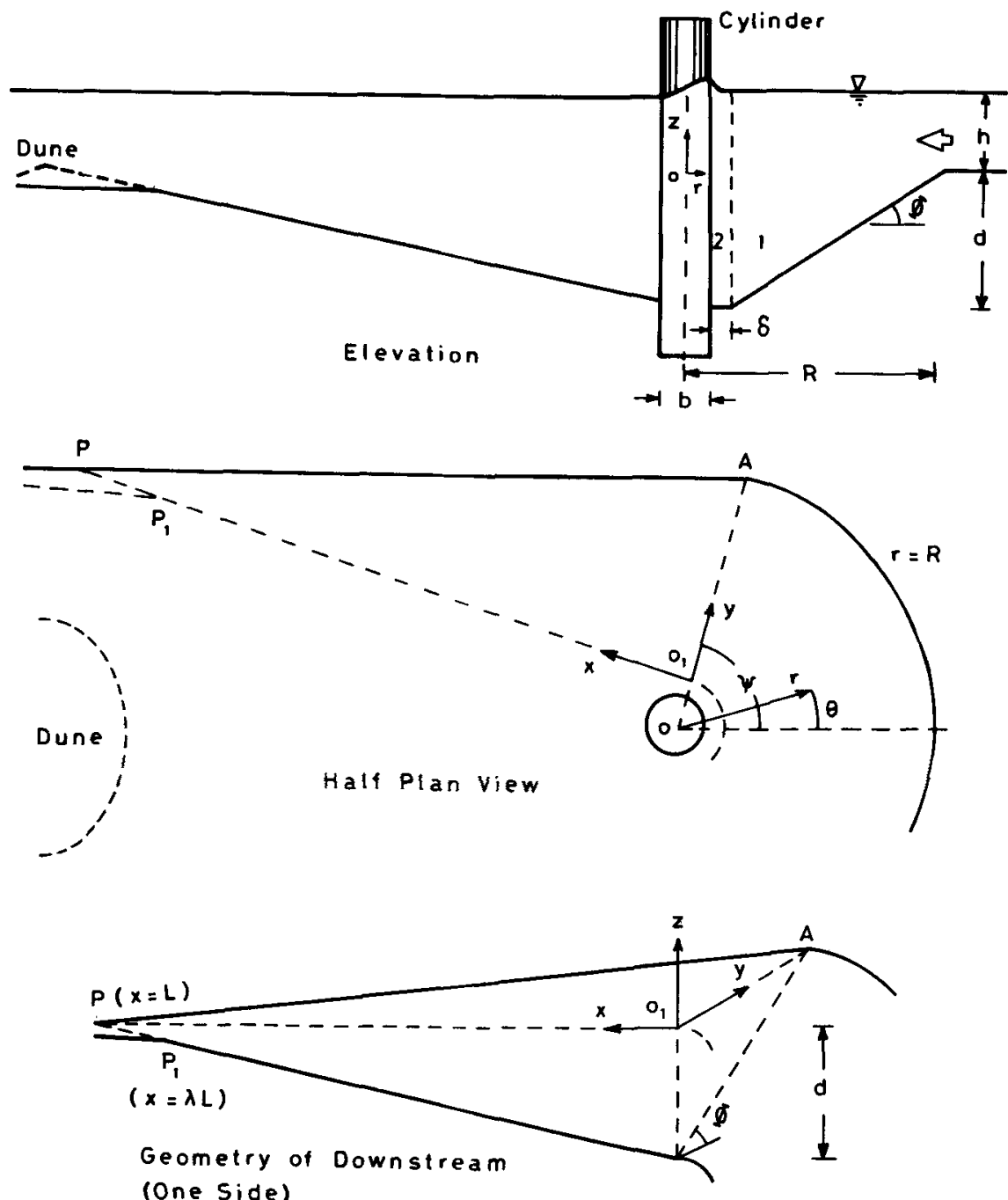

Figure 2. Scour geometry and coordinate systems. 
the flat base adjacent to the cylinder. The edge of the hole was found to be a circular are up to the point of detachment of the horseshoe vortex from the cylinder at $\theta= \pm \psi\left(\approx \pm 75^{\circ}\right)$. The width of zone 2 can be expressed as $\delta=\varepsilon(R-0.5 b)$. where $\varepsilon=\mathrm{a}$ geometric factor, and was found to be 0.1 (Dey 1991); $R$ = radius of the scour hole i.e. $[d /(1-\varepsilon)] \cot \Phi+0 \cdot 5 b ; d=$ depth of scour; and $\Phi=$ dynamic angle of repose. In cylindrical polar coordinates, the quasi-steady velocities in $(\theta, r, z)$ are represented by $\left(u_{u}, v_{u}, w_{u}\right)$.

In the downstream, the measurement was confined to zone 1 , because in zone 2 the flow separation took place to form the wake. $L$, the linear distance from $\mathrm{O}_{1}$ to $P$ (figure 2), can be approximated by $(R-0.5 b)(1-\varepsilon) \tan \psi$. The bed was further washed out at $x \geqslant \lambda . L$ to attain another flatter slope due to an increase in longitudinal velocity with the distance. The factor $\lambda$ was found to be $0.8-0.9$. The quasi-steady velocities in $(x, y, z)$ are denoted by $\left(u_{d}, v_{d}, w_{d}\right)$.

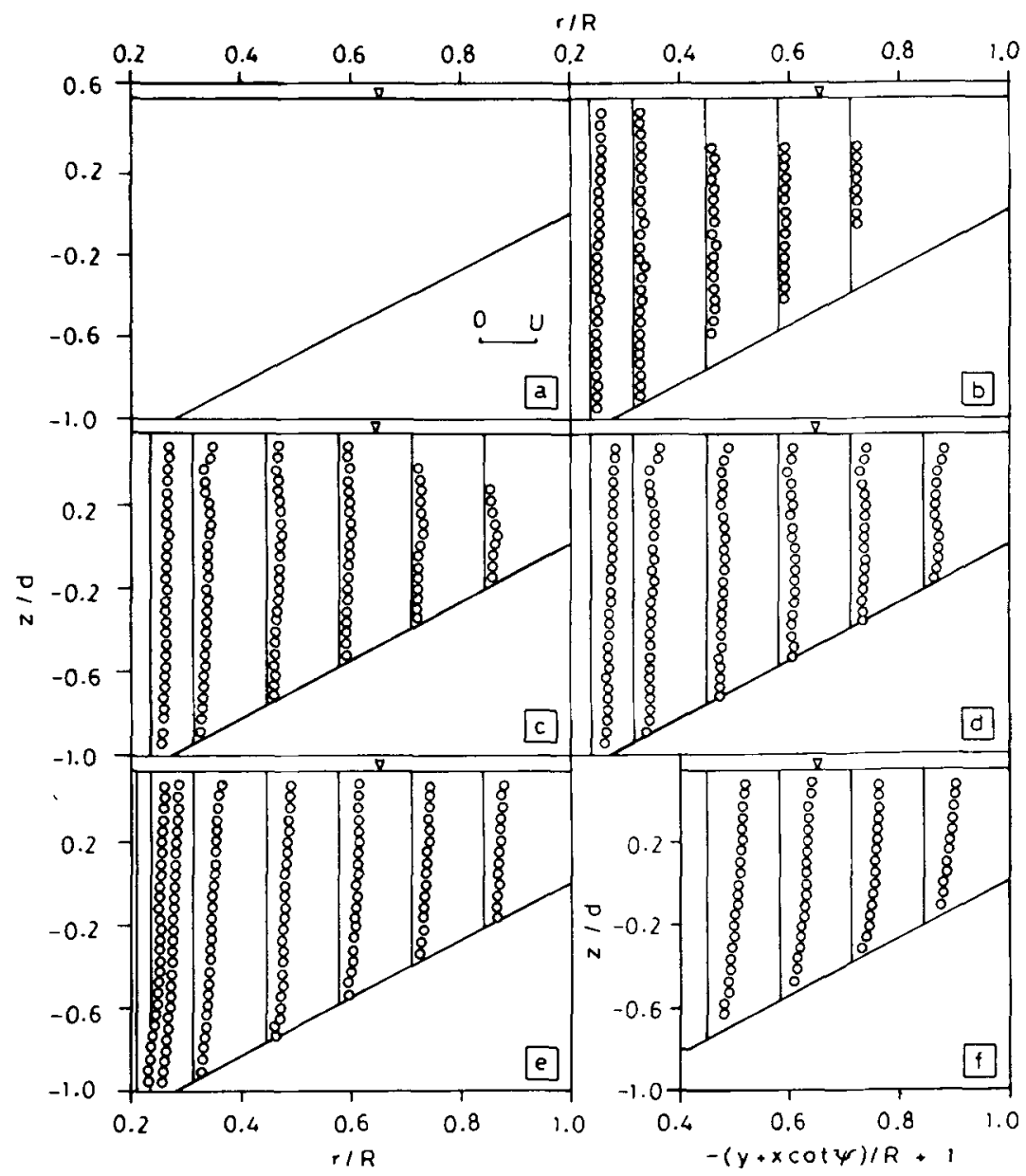

Figure 3. Vertical distributions of $u_{u}$ in the azimuthal planes at (a) $15^{\circ}$, (b) $30^{\circ}$, (c) $45^{\circ}$, (d) $60^{\circ}$ and (e) $75^{\circ}$ upstream; and $u_{d}$ in the transverse plane at (f) $0.2 \mathrm{~L}$ downstream. 


\section{Results}

The velocity measurements were undertaken in the azimuthal planes at $0^{\circ}, 15^{\circ}, 30^{\circ}, 45^{\circ}$, $60^{\circ}$ and $75^{\circ}$ upstream, and in the transverse plane at $0.2 \mathrm{Ldownstream}$. At the upstream edge of the scour hole, the vertical profile of the approaching velocity, reduced considerably due to a larger exposure of flow area ahead, was similar to the profile of the free approaching velocity. The mean velocity at the edge of the hole estimated, comparing the velocity profile at $r=R$ with the profile of the free approaching velocity at $2 \mathrm{~m}$ upstream of the cylinder centre, was $0.8 \mathrm{U}$.

The vertical distributions of normalized tangential component $u_{u} / U$ and longitudinal component $u_{d} / U$ are shown in figure 3 . Here, $u_{u}$ and $u_{d}$ show the characteristic features of the passage of the main flow, as a skewed velocity profile, around the cylinder. The tangential component $u_{u}$ was too small for experimental detection at $15^{\circ}$; and also full detection of $u_{u}$ was not possible at $30^{\circ}$. The distribution of $u_{u}$ is similar to the turbulent flow over a flat surface and can, therefore, be represented by a power law with no-slip at the bed. In zone 2, the passage of downflow by the side of the cylinder enhances $u_{u}$ substantially. The radial distribution of $u_{u}$ is not shown here. However, from a close examination of the vertical distribution of $u_{u}$ it is revealed that there is a considerable skewness in the distribution pattern. This is believed to be due to the pronounced effect of the horseshoe vortex, which is a helical motion like flow, around the cylinder. While fitting the velocity profiles (as a function of normal distance from the bed) by a power law with an exponent $1 / n$, it was found that the ranges of $n$ were $3 \leqslant n \leqslant 6$ for meandering channels (Odgaard 1989) and $7 \leqslant n \leqslant 10$ for straight channels. In the present case, $n$ is found to be approximately $3 \cdot 8$. Hence, the value of $n$ decreases with increase in circulatory motion of vortex flow. Consequently, the skewness of the velocity distribution is more in both radial and vertical directions with the strong circulation in the azimuthal planes. The magnitude of $u_{u}$ is found to be increased with increase in $\theta$, starting with zero at $0^{\circ}$ and becoming a maximum at $75^{\circ}$. In fact, the horseshoe vortex, which originates at $0^{\circ}$, is drifted by the tangential component $u_{u}$ by the side of the cylinder. In the downstream, the longitudinal component $u_{d}$ is similar, in nature, to its preceding upstream part $u_{u}$ with increased magnitude.

The vertical distributions of normalized radial component $v_{u} / U$ are shown in figure 4 . The flow separation at the edge of the scour hole produces a reversal nature of $v_{u}$ in the hole. Thus, the radial component $v_{u}$ changes sign, in the scour hole, on either side of the locus of $v_{u}=0$. This may be compared with the radial velocity in meandering channels (Odgaard 1989). The locus may be assumed to be a median of the triangular section in zone 1 . The vertical distribution of $v_{u}$ varies linearly in the hole, starting with zero at the mid-depth of the hole and becoming a maximum (positive) locally at the bed due to the reversed flow along the slope and a maximum (negative) at $z=0$, with same magnitude. The variation of $v_{u}$ along $z$-axis is exponential above the hole, due to the effect of the approaching flow, starting from the profile at $z=0$ and becoming an overall maximum at the free surface. Although the radial distribution of $v_{u}$ is not shown, a close examination of the vertical distribution reveals a parabolic variation of $v_{u}$ along $r$-axis, starting with zero at the cylinder boundary and becoming a maximum (positive) at the bed following a negative $v_{u}$ on other side of the locus of $v_{u}=0$ in the hole and a maximum (negative) at $r=R$ above the hole. The decreasing nature of $v_{u}$ with increase in $\theta$ is evident. 


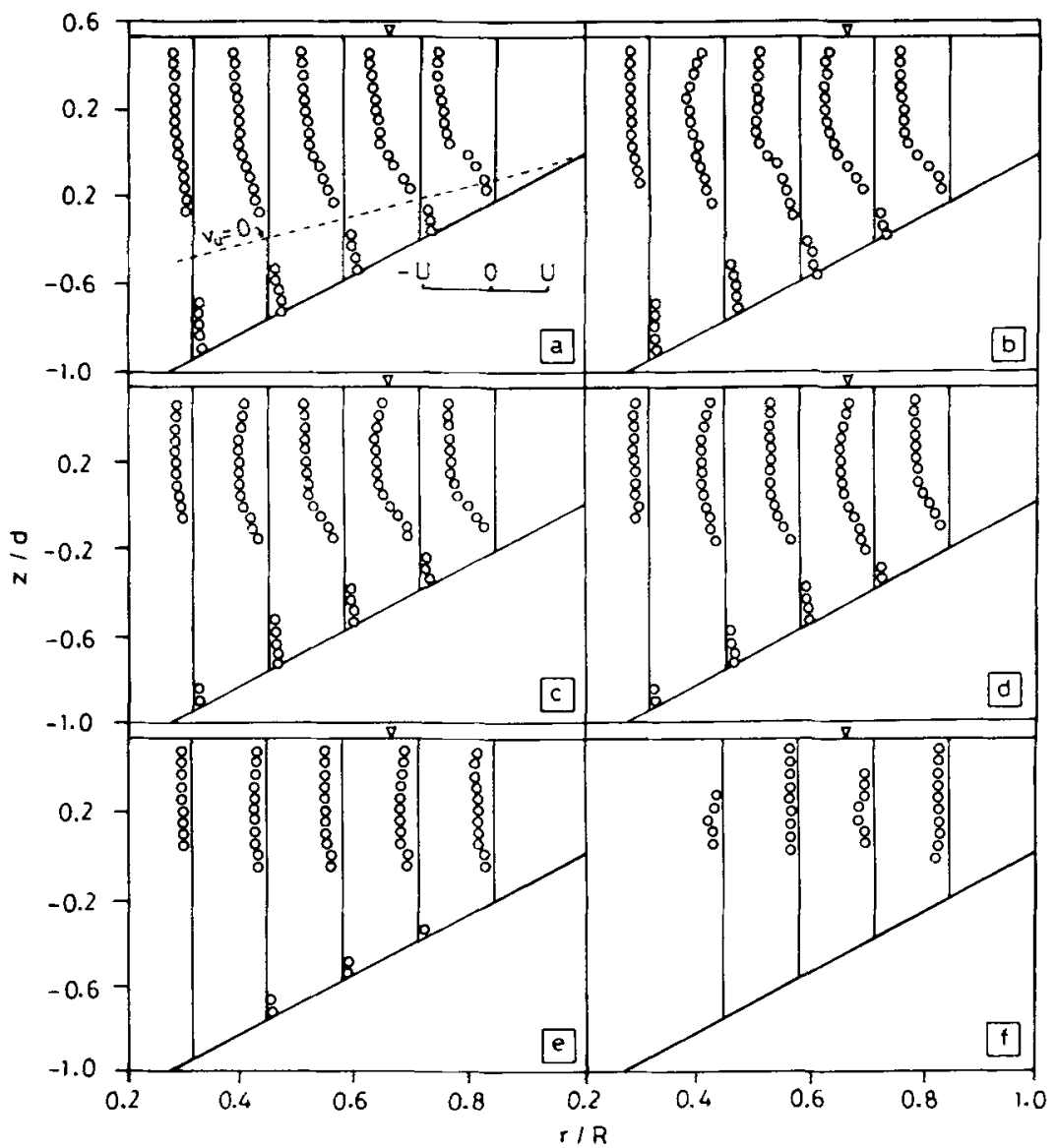

Figure 4. Vertical distributions of $x_{u}$ in the azimuthal planes at (a) 0 , (b) 15 , (c) 30 . (d) 45 , (e) 60 and (f) 75 upstream.

The radial distributions of normalized vertical component $w_{w} / U$ are shown in figure 5. At the edge of the scour hole, the flow separation is also evident from the reversal nature of $w_{u}$ in the hole. The variation of $w_{u}$ is parabolic in the hole, starting with a positive value due to the reversed flow along the sloping bed and becoming a maximum (negative) at the cylinder boundary along $r$-axis and also increasing towards negative value along $z$-axis (revealed from the radial distribution), with opposite signs and a higher magnitude due to the downflow in zone 2 . Locus of $w_{u}=0$ is a curved line meeting at the extreme ends of the sloping bed of the hole. $w_{u}$ changes sign on either side of the locus. At $75, w_{u}$ was very feeble for detection. However, there lies a large helical motion of flow within the scour hole.

In the downstream region, due to the attenuating nature of swirl flow, $\varepsilon_{d}$ and $\boldsymbol{w}_{d}$ were too small to be detected experimentally. The swirl flow loses its identity after travelling a distance of 5 to 6 cylinder diameters. However, the longitudinal velocity component is dominating.

Based on the three-dimensional flow simulation developed by Dey et al (1992a), the flow field around the cylinder in the quasi-equilibrium scour hole was visualized by the 


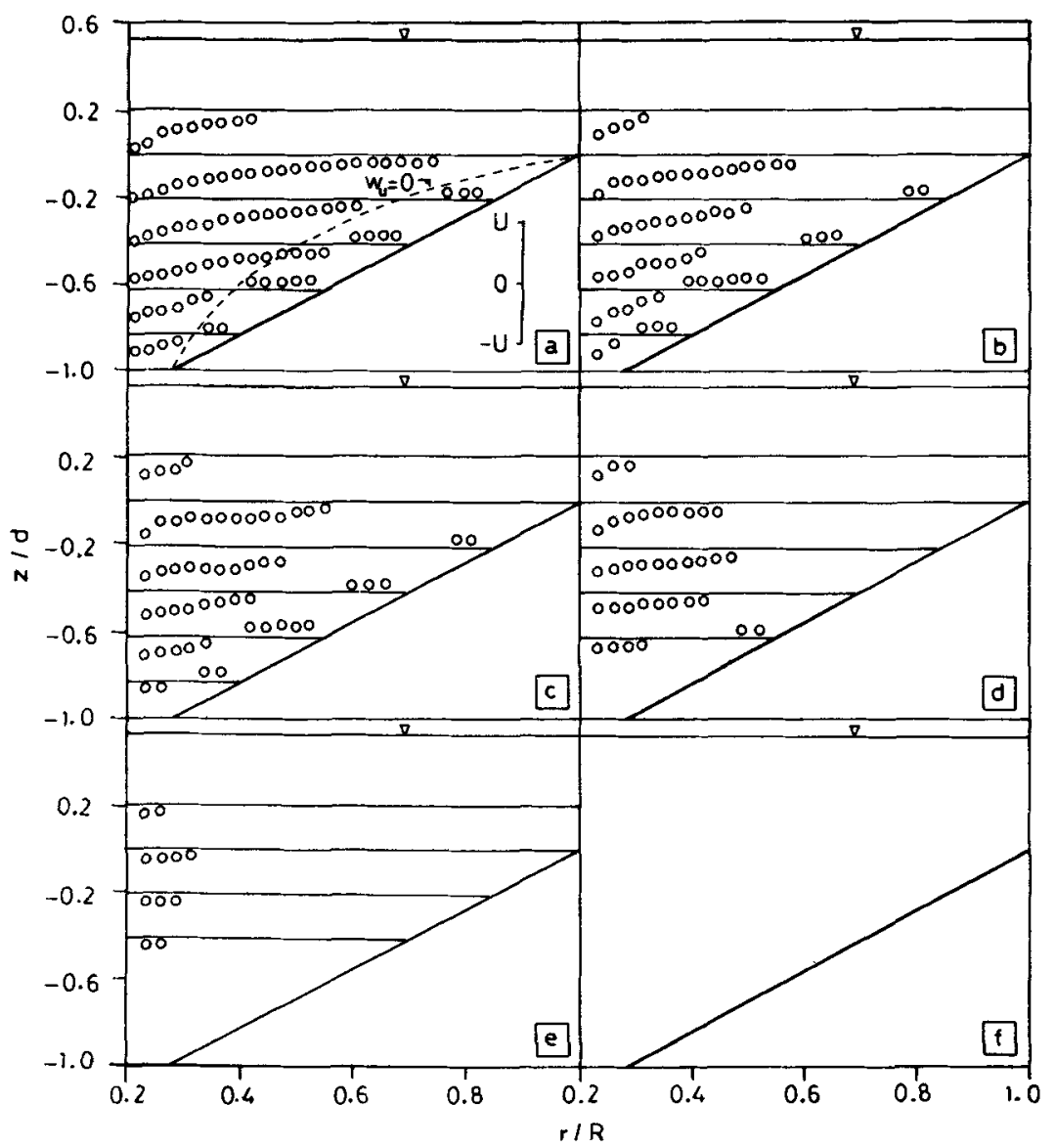

Figure 5. Radial distributions of $w_{u}$ in the azimuthal planes at (a) $0^{\circ}$, (b) $15^{\circ}$, (c) $30^{\circ}$, (d) $45^{\circ}$, (e) $60^{\circ}$ and (f) $75^{\circ}$ upstream.

EGA colour monitor using the measured data. Figure 6 exhibits the velocity vectors in the azimuthal planes. The characteristics of circulatory flow in the scour hole together with the strong downflow along the upstream face of the cylinder are depicted. The circulation is strong along the upstream face and decreases with increase in $\theta$. In $z>0$, the flow is horizontal for $1>r / R \geqslant 0.8$, and then the lower half of the flow gradually curves down and becomes almost vertical adjacent to the cylinder. In contrast, the upper half of the flow is slightly directed upward due to the obstruction caused by the cylinder. However, the flow velocity at the base of zone 2 is about $0.2 U$ in the present case. It was measured by Melville (1975) $0.3 \mathrm{U}$. The result is quite reasonable, because the velocity beneath the horseshoe vortex on flat bed, estimated from the formula given by Qadar (1981), $v_{u}=0.092 b^{-0.5} U^{0.83}$, is $0.4 U$ which reduces considerably in the quasi-equilibrium state due to the expansion of vortex size. The magnitude of total (three-dimensional) velocities around the cylinder in the azimuthal planes are exhibited in figure 7. In absence of $u_{u}$ at $0^{\circ}$, the total velocity is solely circulatory as already shown in figure 6. At $75^{\circ}, u_{u}$ dominates and the circulatory flow becomes weak. In other azimuthal planes, the evolution of the circulatory flow to the exponential distribution of velocity is displayed. The horizontal velocity vectors in the horizontal planes are 

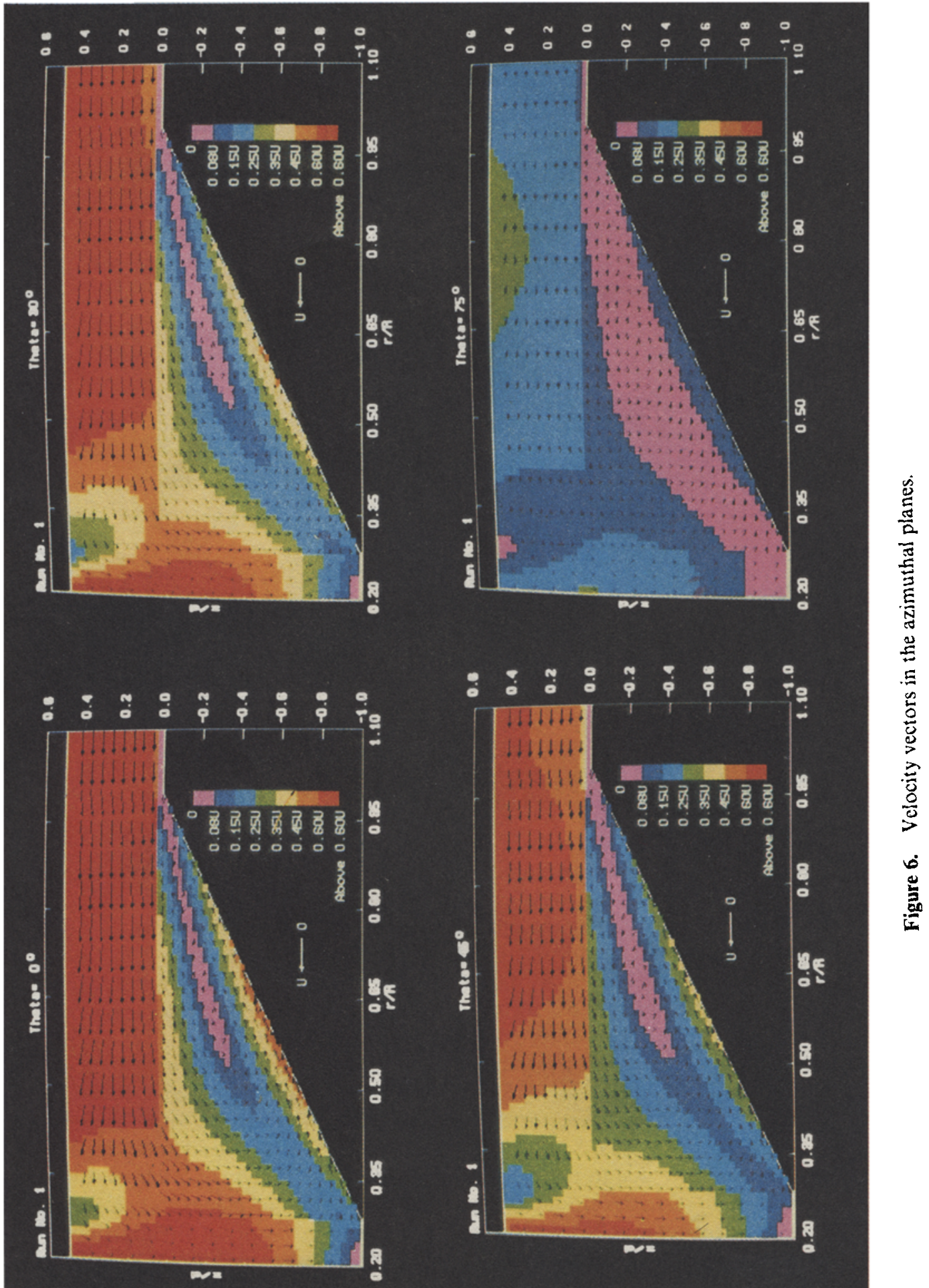

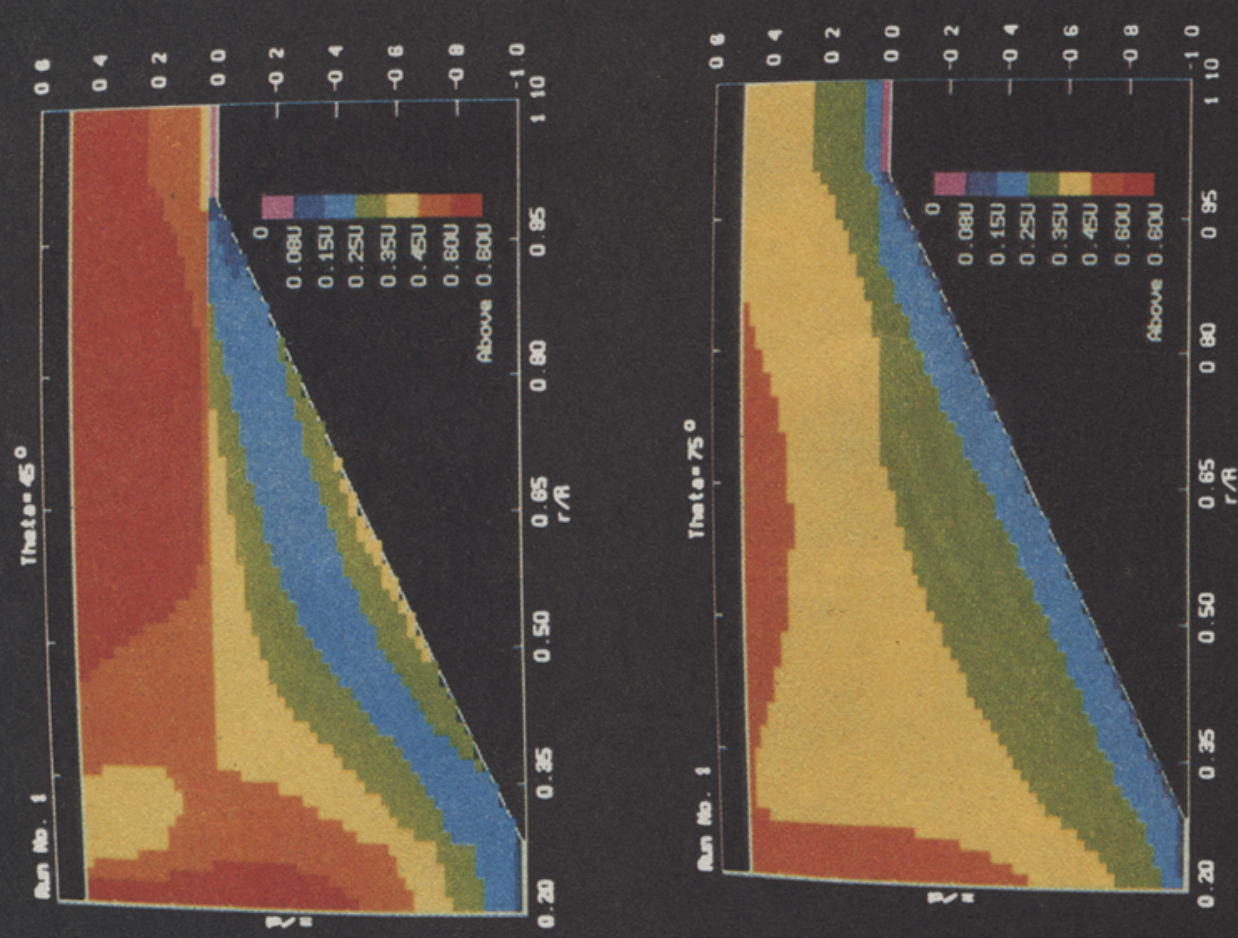

:
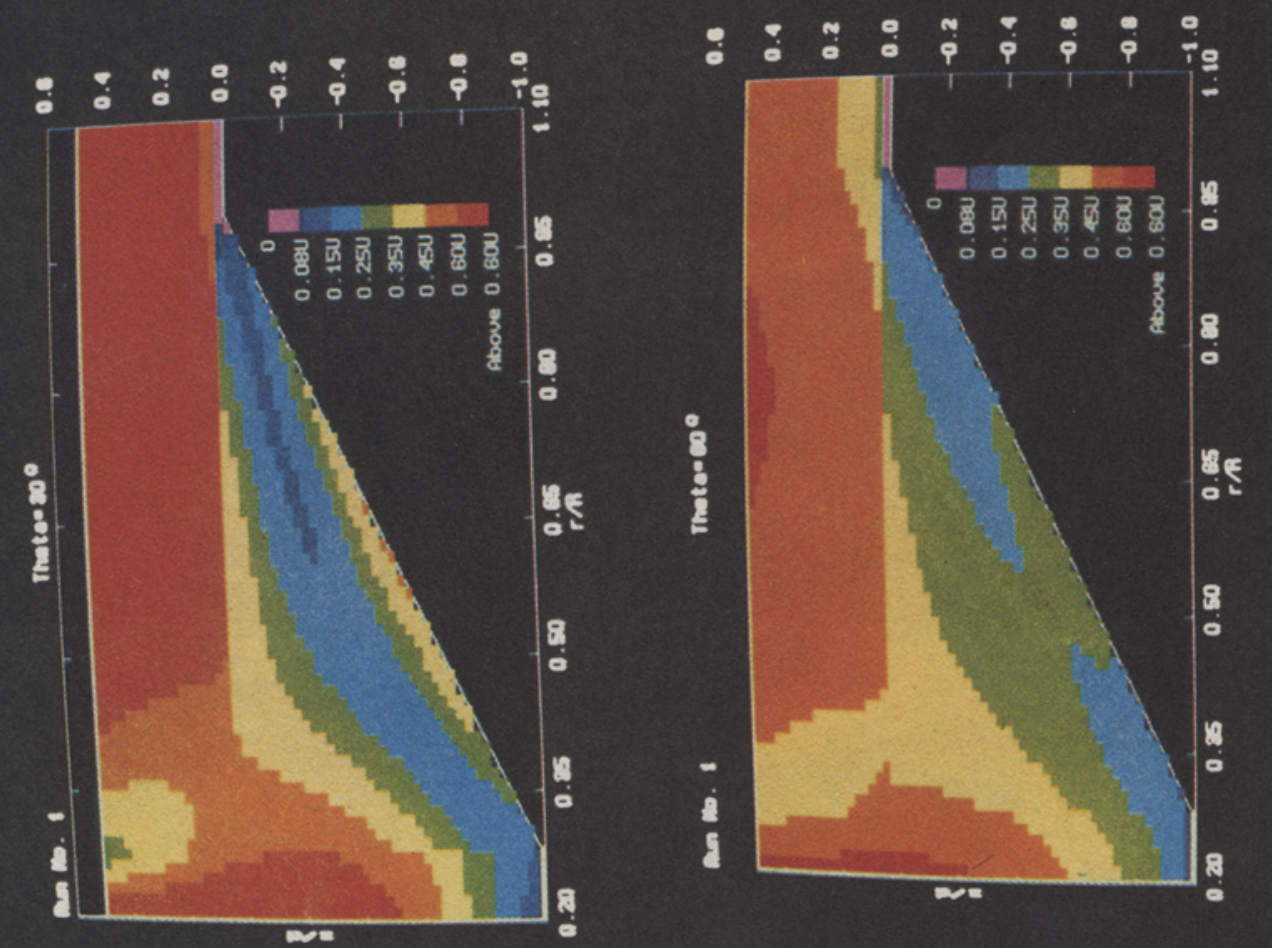

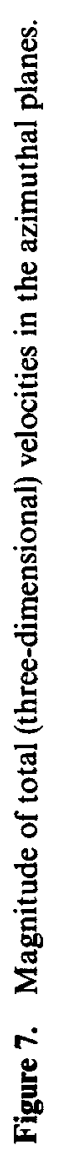



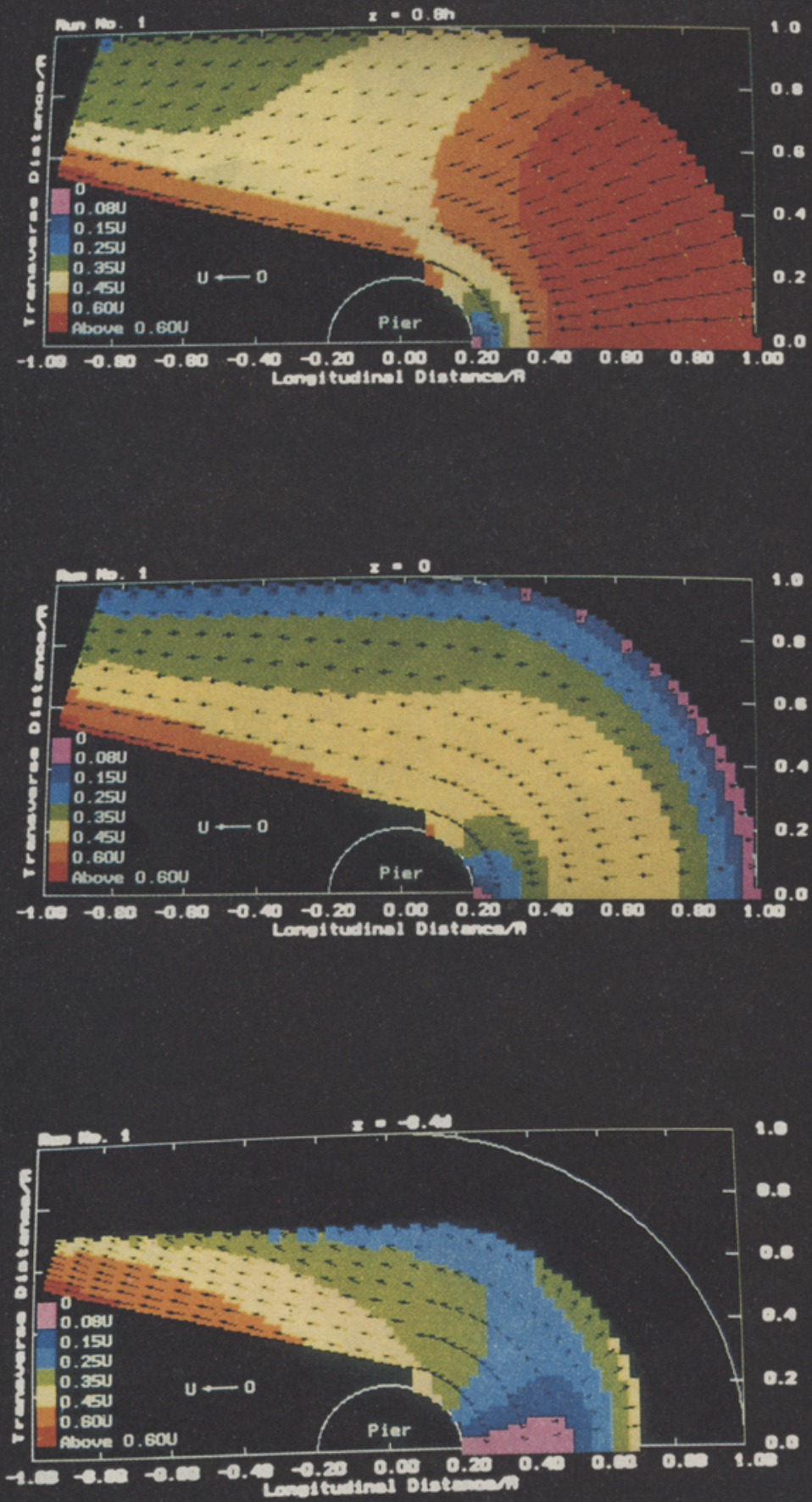

Figure 8. Velocity vectors in the horizontal planes (half plan views). 


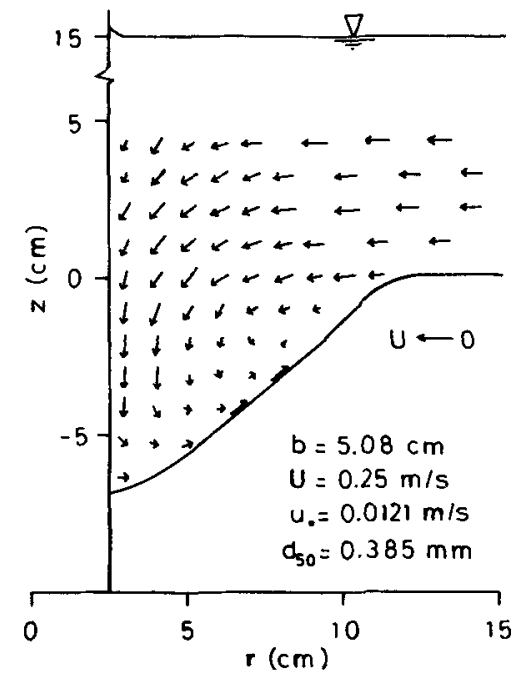

Figure 9. Velocity vectors at $0^{\circ}$ (after Melville \& Raudkivi 1977) used for comparison purposes.

depicted in figure 8 . The horizontal velocity vector at $z=0.8 \mathrm{~h}$ shows the inward motion of the flow. At $z=0$, the flow resembles the irrotational flow past a circular cylinder. The helical motion of flow, in the scour hole, is evident from the flow field at $z=-0 \cdot 4 d$. Figure 9, used for the comparison of a part of the present data, shows the observations of Melville \& Raudkivi (1977) at $0^{\circ}$ under equilibrium scour. Their observations agree with the flow characteristics at $0^{\circ}$ presented in figure 6 , which establishes the validation of the present measuring technique.

The physics of the flow is analyzed with the help of the above results. The main characteristic features of the flow around a cylinder are a relatively large secondary vortex flow and skewed velocity distributions. The approaching flow separates at the upstream edge of the scour hole, known to be a line of separation, to form a vortex flow in the hole which acts as a zone of separation. The limiting streamlines along the original bed upstream (due to the approaching flow) and the sloping bed (due to the reversed flow) merge at the edge of the scour hole forming separated streamlines. Thus, a surface of separation is produced in the form of an envelope. In this process, the approaching flow curves down into the hole and rolls to form a vortex flow which migrates downstream by the sides of the cylinder.

\section{Concluding remarks}

The experimental results of the distribution patterns of velocity components for a three-dimensional vortex flow around a circular cylinder in a quasi-equilibrium scour hole under a clear water regime have been presented. In the upstream, the tangential component $u_{u}$ follows a power law, starting with zero at the bed and becoming a maximum at the free surface and at the cylinder boundary, with a higher magnitude in zone 2 . The radial component $v_{u}$ varies linearly in the hole along $z$-axis, starting with zero at the mid-depth of the scour hole and becoming a maximum locally at the bed and at $z=0$, with opposite signs and same magnitude. $v_{u}$ varies exponentially above the hole along $z$-axis, starting from the profile at $z=0$ and becoming an overall maximum 
at the free surface. Along $r$-axis, the distribution of $v_{u}$ is parabolic, starting with zero at the cylinder boundary and becoming a maximum at the bed in the hole and at $r=R$ above the hole. The variation of vertical component $w_{u}$ is parabolic in the hole. starting with a positive value at the bed and becoming a maximum at the cylinder boundary along $r$-axis and at $z=0$ along $z$-axis, with opposite signs and a higher magnitude in zone 2 . In the downstream, the attenuating nature of swirl flow becomes very feeble and the longitudinal component $u_{d}$ becomes gradually stronger than its preceding part $u_{u}$ upstream. The flow simulation results show a helical motion of fluid around the cylinder.

This investigation is a part of the doctoral dissertation work by the author in the Department of Civil Engineering. Indian Institute of Technology, Kharagpur under the joint supervision of S K Bose and G L N Sastry. The helpful suggestions from S C Jain, Institute of Hydraulic Research, University of Iowa, are gratefully acknowledged. The author also appreciates the assistance provided by B Dey during the investigation.

\section{List of symbols}

$\begin{array}{ll}b & \text { cylinder diameter; } \\ d & \text { quasi-equilibrium depth of scour; } \\ d_{16}, d_{50}, d_{84} & 16,50,84 \% \text { finer particle diameters; } \\ h & \text { approaching flow depth; } \\ L & \text { longitudinal extension of scour hole downstream; } \\ n & \text { exponent; } \\ R & \text { radius of scour hole; } \\ U & \text { mean approaching velocity; } \\ u_{u}, v_{u}, w_{u} & \text { velocities in cylindrical polar coordinates upstream; } \\ u_{d}, v_{d}, w_{d} & \text { velocities in Cartesian coordinates downstream; } \\ u_{*} & \text { shear velocity; } \\ u_{* c} & \text { critical shear velocity; } \\ x, y, z & \text { Cartesian coordinates; } \\ \varepsilon, \lambda & \text { factors; } \\ \delta & \text { width of zone 2; } \\ \phi & \text { angle of repose; } \\ \Phi & \text { dynamic angle of repose; } \\ \theta, r, z & \text { cylindrical polar coordinates; } \\ \psi & \text { angle of detachment of horseshoe vortex; } \\ \sigma_{g} & \text { geometric standard deviation. }\end{array}$

\section{References}

Breusers H N C, Nicollet G, Shen H W 1977 Local scour around cylindrical piers. J. Hydraul. Res. 15: 211-252

Dargahi B 1982 Local scouring around bridge piers - A review of practice and theory. Bulletin No. 114, Hydraulics Lab, Royal Inst. Technol., Stockholm 
Dargahi B 1987 Flow field and local scouring around a cylinder. Bulletin No. 137, Hydraulics Lab, Royal Inst. Technol., Stockholm

Dey S 1991 Clear water scour around circular bridge piers: $A$ model. $\mathrm{Ph} \mathrm{D}$ thesis, Indian Inst. Technol., Kharagpur

Dey S, Bose S K, Sastry G L N 1992a Clear water scour at circular piers, part I: Flow model. Proc. 8th Cong. IAHR Asian and Pacific Div. IIIC: 69-80

Dey S, Bose S K, Sastry G L N 1992 b Clear water scour at circular piers, part II: Design formula. Proc. 8th Cong. IAHR Asian and Pacific Div. IIIC: 81-92

Goldstein R J 1983 Fluid mechanics measurements (New York: Hemisphere)

Hjorth P 1975 Studies on the nature of local scour. Bulletin. Series A No. 46, Dept. of Water Resources Eng., Univ. of Lund

Jain S C, Fischer E E 1979 Scour around circular piers at high Froude numbers. Report No. FHWA-RD-79-104, US Dept. of Transportation, Federal Highway Administration, Washington, $\mathrm{DC}$

Melville B W 1975 Local scour at bridge sites. Report No. 117, Univ. of Auckland

Melville B W, Raudkivi A J 1977 Flow characteristics in local scour at bridge piers. J. Hydraul. Res. 15: 373-380

Nwachukwu B A 1979 Flow and erosion near groyne like structures. $\mathrm{Ph} D$ thesis, University of Alberta

Odgaard A J 1989 River-meander model I: Development. J. Hydraul. Eng., Am. Soc. Civ. Eng. 115: $1433-1450$

Qadar A 1981 The vortex scour mechanism at bridge piers. Proc. Inst. Civ. Eng. 71: 739-757

Rajaratnam N, Muralidhar D 1967 Yaw and pitch probes. Hydraul. Instrum. Series, Dept. Civ. Eng., University of Alberta

Raudkivi A J 1986 Functional trends of scour at bridge piers. J. Hydraul. Eng. Am. Soc. Civ. Eng. 112: $1-13$

Shukry A 1950 Flow around bends in open flumes. Trans. Am. Soc. Civ. Eng. 115: 715-779

Tingsanchali T, Maheswaran S 1990 2-D depth-averaged flow computation near groyne. $J$. Hydraul. Eng., Am. Soc. Civ. Eng. 116: 71-86 